\title{
Conociendo otros sectores: La óptica en España
}

\section{MARGARITA BARRERA LOZANO}

Universidad de Sevilla, Fctd. CC.EE. y EE., Avda. de Ramón y Cajal, 1, 41018 Sevilla, España. Email: mbarrera3@us.es

\section{RESUMEN}

Este trabajo tiene como objetivo cuantificar la estructura de la industria de la óptica oftálmica en España, con la finalidad de conocer el funcionamiento y crear un marco de referencia para el estudio del sector.

Para la realización de dicho trabajo se ha hecho uso de fuentes de datos primarios y secundarios que recogen la cuantificación y el tamaño del sector y la segmentación del mismo.

La industria objeto de análisis muestra una alta versatilidad ante las variaciones en la coyuntura económica y un alto valor añadido de ciertas actividades. La diferenciación por segmentos demuestra características singulares en el comportamiento de la línea de lentes frente a la de gafas en estructura empresarial y, especialmente, de la línea de lentes de contacto.

Palabras clave: análisis sectorial, sector óptico, cuantificación económica

\section{Analyzing sectors: Optics in Spain}

\begin{abstract}
This work aims to quantify the structure of the ophthalmic optical industry in Spain, with the objective to ascertain the functioning and to create a reference framework for the analysis of this sector.

To develop this work, primary and secondary datum has been used, covering the quantification and characteristics of the sector.

The core of this analysis shows the sector to be highly versatile to variations in the economic situation, and a high value added concentrate in specific activities among those included in the sector. Economic segments are demonstrated to be remarkably different, with a singular behaviour especially in contact lenses enterprise structure, in contrast to that of spectacle frames.
\end{abstract}

Keywords: Sectoral Analysis, Optical Sector, Economic Assessment

Clasificación JEL: D29, D43, L10, L50, L69

Artículo recibido en junio de 2017 y aceptado en diciembre de 2017

Artículo disponible en versión electrónica en la página www.revista-eea.net, ref. ə-36201 


\section{INTRODUCCIÓN}

El sector óptico abarca aquellas actividades económicas que se basan principalmente en el suministro de productos ópticos, englobando un amplio espectro de actividades y tipos de productos. La óptica oftálmica se refiere a aquella parte de la óptica que mantiene relación con la compensación o protección de la visión, por lo que el sector de la óptica oftálmica comprende aquellas empresas que se dedican a la provisión de lentes y gafas. La escasez de estudios al respecto, hace que nos centremos en el análisis del sector industrial. Por lo tanto, este trabajo se centrará en las empresas industriales de: lentes oftálmicas, lentes de contacto y monturas y gafas de sol.

Una de las principales aportaciones de este trabajo es la cuantificación económica del nivel de producción y distribución de importaciones, desentrañando las diferencias por segmentos. Al contrario que las grandes ramas de actividad que componen la economía, u otros sectores de gran tamaño, el sector óptico raramente ha sido analizado, encontrando trabajos esencialmente centrados en el comercio minorista.

Los trabajos de referencia sobre el sector son las realizadas por la Federación de empresas de óptica (FEDAO), los denominados Libros Blancos de la Visión (Visión y Vida, 2003; 2006; Visión y Vida y FEDAO, 2009; 2013), que recogen información sobre el mercado español de óptica oftálmica y la demanda final. Relacionado con los anteriores, también destacan los estudios sectoriales del comercio en ópticas de Gómez (2008; 2009; 2010; 2011; 2012; 2013), que ofrece información por productos sobre la facturación de los establecimientos de óptica en la economía española. Asimismo, también existen informes de consultoría, como los realizados por DBK (2010; 2012; 2015; 2017), que llevan a cabo un análisis en la misma línea que los referidos anteriormente, aunque detallando marcas y empresas.

Trabajos a nivel empresarial, también de establecimientos de óptica, se encuentran en los desarrollados por Cegarra \& Sánchez Polo (2001a) y Rodrigo Moya \& Cegarra (2003) que analizan la organización sectorial y la tipología de las ópticas, mientras que Martínez \& Monserrat (2010) se centran en las del tipo franquiciado. Cegarra Navarro \& Sánchez Polo (2001b) (clasificación indicada en la Tabla 1), comienzan a analizar las estrategias comerciales en los establecimientos de óptica, que posteriormente se amplía por otros estudios sobre la fidelización del cliente (Cegarra Navarro \& Rodrigo Moya, 2003) (cuyo análisis se resumen en la Tabla 2), sobre el aprendizaje relacional en las ópticas (Dewhurst \& Cegarra-Navarro, 2004; Cegarra-Navarro, 2004; 2007; Cegarra-Navarro \& Rodrigo-Moya, 2005; Cegarra-Navarro \& Dewurst, 2006) y el des-aprendizaje en la interacción entre la organización y el cliente final (Cegarra-Navarro \& Sánchez-Polo, 2008). 
Tabla 1

Estrategias comerciales de las ópticas

\begin{tabular}{|l|c|}
\hline \multirow{2}{*}{ Estrategia defensiva } & Disminuir los motivos de descontento \\
\cline { 2 - 2 } & Mejora de la calidad \\
\cline { 2 - 2 } & Servicio al consumidor \\
\hline Estrategia ofensiva & Fuerte relación entre el cliente y la óptica: Sentimiento de comunidad \\
\hline
\end{tabular}

Fuente: Elaboración propia. Cegarra Navarro \& Sánchez Polo (2001b).

Tabla 2

Tipología de las ópticas

\begin{tabular}{|c|c|c|c|}
\hline Criterio & \multicolumn{2}{|c|}{ Tipología } \\
\hline Por producto y servicio & $\begin{array}{c}\text { Lentes de Contacto y } \\
\text { Ayudas de Baja Visión }\end{array}$ & \multicolumn{2}{|c|}{ Monturas y audífonos } \\
\hline Por antigüedad & $\begin{array}{c}\text { Muy jóvenes (menos de } \\
\text { 4años) }\end{array}$ & Jóvenes (4-9 años) & $\begin{array}{c}\text { Maduras (más de } \\
9 \text { años) }\end{array}$ \\
\hline $\begin{array}{c}\text { Por tamaño de empresa (número } \\
\text { de trabajadores) }\end{array}$ & $\begin{array}{c}\text { Micro (menos de 9) } \\
\text { Pequeña (10 -49) }\end{array}$ & Mediana (50-249) \\
\hline Propiedad por parte del gerente & Coincidente & \multicolumn{2}{|c|}{ No coincidente } \\
\hline Estabilidad laboral & $\begin{array}{c}\text { Menos del 80\% de la } \\
\text { plantilla fija }\end{array}$ & $\begin{array}{c}\text { Más del 80\% de la } \\
\text { plantilla fija }\end{array}$ & \\
\hline Edad del gerente & Menos de 33 años & 33-40 años & Más de 40 años \\
\hline Titulación específica del gerente & $\begin{array}{c}\text { Diplomado en Óptica y } \\
\text { Optometría }\end{array}$ & Otra & \multicolumn{2}{|c|}{ No } \\
\hline Existencia de grupos de trabajo & Si & \multicolumn{2}{|c|}{} \\
\hline
\end{tabular}

Fuente: Elaboración propia. Rodrigo Moya \& Cegarra (2003).

Sin embargo, la composición y la estructura de la rama industrial del sector de la óptica oftálmica han permanecido ajenas al análisis económico académico hasta el planteamiento metodológica para su desagregación en la economía española (Barrera, Causapé, \& Vallés, 2015). Además de este documento, sólo se dispone de estudios de consultoría, como los realizados regularmente por DBK (2009; 2011; 2014; 2016; 2017). Esta circunstancia destaca la importancia del trabajo que aquí se presenta y que presenta la estructura económica y características principales de la industria de la óptica, para su futuro desarrollo analítico.

Con el objetivo de conocer las características del sector y desarrollar su estructura, evolución y contexto, este trabajo presenta el marco estadístico de referencia y el procedimiento de análisis. Posteriormente, expone la estructura de la industria óptica oftálmica, su evolución reciente y el marco internacional. Finalmente procede a la discusión y conclusiones del trabajo.

\section{CLASIFICACIÓN SECTORIAL Y FUENTES DE DATOS UTILIZADAS}

La clasificación utilizada como referencia en gran parte de los análisis 
sectoriales es la nomenclatura general de actividades económicas de las Comunidades Europeas (NACE) (Eurostat, 2006), a partir de la cual se desarrolla la clasificación nacional de actividades económicas (CNAE), ambas siguen nomenclátores semejantes a la clasificación por productos (CPA). Un mayor desglose para productos industriales se encuentra en la clasificación de producción de canteras, minería y manufacturas (Prodcom). Son las Revisiones 1 y 2 de la NACE, las 93 y 09 de la CNAE y la CPA-2008 las más utilizadas actualmente, aunque, para determinada información la Prodcom es la que mayor desagregación ofrece. También es relevante conocer los códigos impositivos de los productos, a través del impuesto de actividades económicas (IAE), para la identificación de empresas en las bases de datos que no ofrecen suficiente desagregación a través de las clasificaciones de actividades.

Tabla 3

Códigos estadísticos de la óptica oftálmica en las actividades económicas y en los productos

\begin{tabular}{|c|c|}
\hline Clasificación & Códigos \\
\hline CNAE-93 & 33.40.1: Fabricación de lentes correctoras de la visión \\
\hline CNAE-09 & 32.50: Fabricación de instrumentos y suministros médicos y odontológicos \\
\hline CPA-08 & $\begin{array}{l}\text { 32504: Gafas, lentes y sus componentes } \\
\text { 325041: Lentes de contacto; lentes de cualquier material para gafas } \\
\text { 325042: Gafas correctoras, protectoras u otras, y artículos similares } \\
\text { 325043: Monturas de gafas o artículos similares }\end{array}$ \\
\hline Prodcom & $\begin{array}{c}\text { 32.50.41.30: Lentes de contacto } \\
\text { 32.50.41.53: Lentes no correctoras para gafas, sin montar } \\
\text { 32.50.41.55: Lentes correctoras para gafas, trabajadas completamente en ambas caras, } \\
\text { monofocales, sin montar } \\
\text { 32.50.41.59: Lentes correctoras para gafas, trabajadas completamente en ambas caras, } \\
\text { sin montar, excepto monofocales } \\
\text { 32.50.42.50: Gafas de sol } \\
\text { 32.50.42.90: Gafas correctoras, protectoras y similares, excepto de sol } \\
\text { 32.50.43.50: Monturas de gafas o artículos similares, de plástico } \\
\text { 32.50.43.90: Monturas de gafas o artículos similares, excepto de plástico }\end{array}$ \\
\hline IAE & $\begin{array}{l}\text { 393.1: Instrumentos ópticos y material fotográfico y cinematográfico (excepto monturas } \\
\text { para gafas) } \\
\text { 393.2: Fabricación de monturas para gafas (excepto las de plástico) }\end{array}$ \\
\hline
\end{tabular}

Fuente: Elaboración propia. INE (2003; 2012); Eurostat (2008; 2012); Real Decreto Legislativo 1175/1990, de 28 de septiembre, por el que se aprueban las tarifas y la instrucción del Impuesto sobre Actividades Económicas. Texto consolidado (2013); Agencia Tributaria (2015).

En este documento nos referiremos a CNAE-2009 y a la Prodcom correspondiente, que en este caso es la Revisión 2, cuyos códigos se observan en la Tabla 3. Sin embargo ninguna de ellas permite desentrañar los datos referentes al sector óptico a nivel empresarial con suficiente flexibilidad como para poder conocer su estructura. Es por esta razón por la que se produce al análisis exhaustivo de empresas, y se recurre a los datos contables de las mismas a través de sus cuentas, recurriendo a la base de datos Sabi de Bureau Van Dijks. 
El primer paso para proceder a la cuantificación de las empresas es determinar el número de empresas que la componen. El dato más accesible es aquel del número de asociados a la Asociación de fabricantes e importadores de óptica oftálmica, que asciende ${ }^{1}$ a 36 empresas. Un análisis más exhaustivo se desarrolla partiendo de la búsqueda exploratoria de empresas a través de bases de datos de marketing ${ }^{2}$. Posteriormente, se procede a determinar el objeto social de las empresas a través de los datos contenidos en el formato estándar de Sabi y de la información disponible en las páginas web.

Una vez determinada la actividad específica de las empresas y su participación en la actividad de óptica se procede a determinar los requisitos de inclusión de empresas como parte del sector. Estos requisitos se componen de cinco condiciones suficientes de inclusión y una condición suficiente para la exclusión, más una condición necesaria: que existan indicios de su actividad dentro del sector de la óptica oftálmica a través de la información previamente obtenida. Los requisitos son los siguientes:

Condiciones suficientes de inclusión:

1. Que perteneciese a la CNAE correspondiente a óptica oftálmica y se indicase dicha actividad en el objeto social.

2. Que en su página web se indicase que la empresa llevaba su actividad en este ámbito.

3. Que perteneciese al IAE correspondiente a fabricación de óptica oftálmica.

4. Que en la descripción de la actividad que se ofrecen en las cuentas de la empresa apareciese la de fabricación y/o importación o exportación de algunos de los productos considerados de óptica oftálmica.

5. Que se tratase de empresa global en el campo de la óptica oftálmica y de prestigio internacional con actividad conocida en la fabricación de productos de óptica oftálmica.

Condición suficiente de exclusión:

- Que, cumpliéndose alguna de las cuatro primeras condiciones de inclusión, perteneciese a unas de las siguientes clases de CNAE: comercio al por menor o actividades previas o posteriores a las manufacturas de óptica oftálmica en la cadena de suministro, como por ejemplo la 25: Fabricación de productos metálicos, excepto maquinaria y equipo, por entenderse que la actividad sea la de fabricación de partes de gafas y no de gafas en sí o la 95: Reparación de ordenadores, efectos personales y artículos de uso doméstico, así como las de promoción inmobiliaria y similares.

\footnotetext{
${ }^{1}$ Consultado en 2014.

${ }^{2}$ En concreto Guia Puntex e infoóptica.
} 
Una vez creado el universo de empresas a estudiar, se procede también a ponderar la participación de cada empresa en la actividad correspondiente de óptica oftálmica, a través de la información pública de la empresa, su información contable en las notas de los depósitos de cuentas o contactando directamente con las empresas cuando dichas opciones no ofrecen claridad.

\section{ESTRUCTURA DEL SECTOR DE LA ÓPTICA OFTÁLMICA}

La estructura sectorial, considerando las empresas socias de AEO, mantienen una distribución equitativa entre los tipos de producción que se diferencian en el sector en el siguiente orden: lentes oftálmicas, gafas y lentes de contacto, ascendiendo a un total de 36 empresas entre fabricantes e importadoras.

Una vez se ha desarrollado el análisis exploratorio de empresas e incluido aquellas que cumpliesen los criterios fijados, encontramos un total de 53 empresas $^{3}$ en el ejercicio $2012^{4}$, las que compondrían el sector de manufacturas de óptica oftálmica compuesto por empresas fabricantes y globales.

El empleo total de las empresas del sector alcanza 4.000 empleos, de los que 2.500 corresponden a óptica oftálmica, y el resto, principalmente, a la industria farmacéutica.

Figura 1

Composición del sector considerando empresas fabricantes

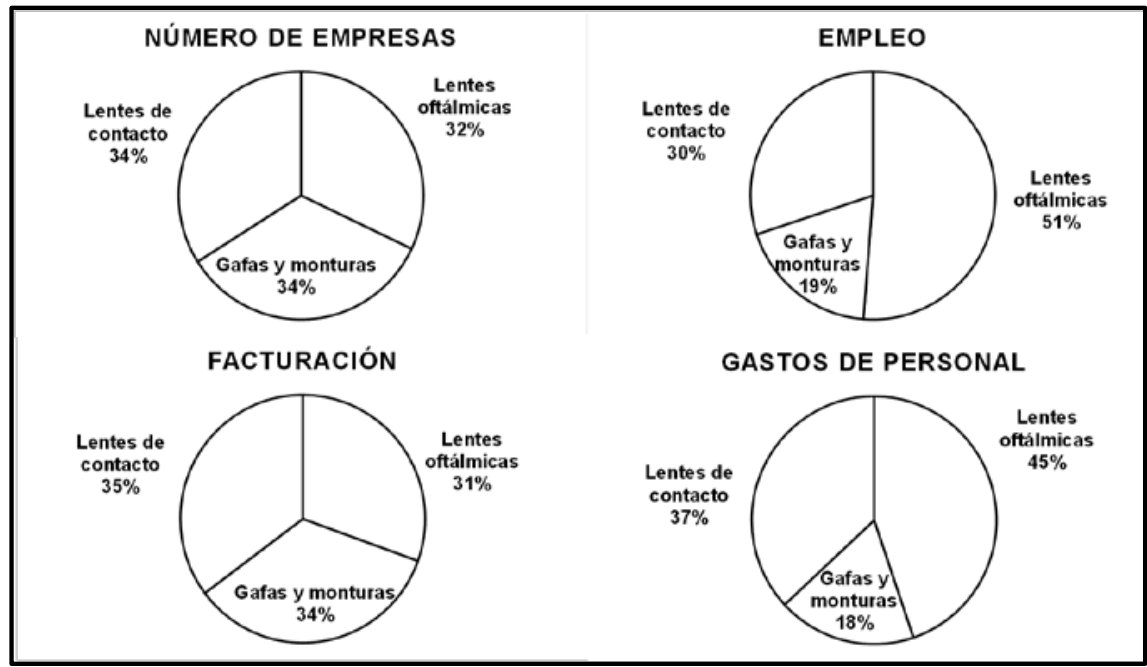

Fuente: Elaboración propia, Sabi (2014).

\footnotetext{
${ }^{3}$ Habiendo eliminado aquellas empresas de las que se tenía constancia de su extinción.

${ }^{4}$ Año para el que se realizó dicha consulta, y que se elige por ser aquel para el que se dispone de mayor información en momento de la búsqueda.
} 
La Figura 1 representa información acerca de aquellas empresas que participan de uno u otro modo en la actividad manufacturera, y corresponde a datos recabados en 2014, por lo que el año de referencia es el 2012. En ella se observa que la participación de cada segmento de actividad varía en número de empresas, número de empleados, facturación o gastos de personal. Aunque, tanto el número de empresas como la facturación son semejantes entre cada una de ellas, aquellas con mayor valor añadido y empleo creado son las actividades de fabricación de lentes oftálmicas seguidas de lentes de contacto. Lo que remarca la diferente estructura en cada una de las actividades.

Estas diferencias existen también en el tamaño empresarial, como se deriva de la Tabla 4 que muestra el número de empresas por número de empleados. Un $57 \%$ de las empresas fabricantes de óptica oftálmica tienen más de 20 empleados. Distinguiendo el tipo de actividad, se encuentra que la mayoría de las empresas de monturas y gafas, un 61\%, tienen menos de 20 empleados. Siendo los porcentajes de empresas mayores de 20 empleados de lentes oftálmicas y lentes de contacto, respectivamente un 59\% y un $72 \%$. Por lo que la escala de producción más competitiva es aquella de lentes de contacto.

Tabla 4

Tamaño de las empresas fabricantes de óptica oftálmica

\begin{tabular}{|c|c|c|c|c|}
\hline & $\begin{array}{c}\text { Menos de 20 } \\
\text { empleados }\end{array}$ & $\begin{array}{c}\mathbf{2 0 - 5 0} \\
\text { empleados }\end{array}$ & $\begin{array}{c}\mathbf{5 0 - 1 0 0} \\
\text { empleados }\end{array}$ & $\begin{array}{c}\text { Más de 100 } \\
\text { empleados }\end{array}$ \\
\hline Lentes oftálmicas & 7 & 5 & 1 & 4 \\
\hline Monturas y gafas & 11 & 6 & 0 & 1 \\
\hline Lentes de contacto & 5 & 5 & 3 & 5 \\
\hline
\end{tabular}

Fuente: Elaboración propia. Sabi (2014). Datos en número.

Las diferencias encontradas entre las actividades relacionadas con lentes y las relacionadas con monturas, junto al crecimiento del número de empresas distribuidoras de monturas y gafas importadas, llevan a plantear una nueva búsqueda que incluya este último tipo de empresas. Como resultado, las empresas estudiadas pasan a componerse por empresas fabricantes e importadoras, independientemente de que desarrollen o no actividad a nivel global. Fruto de la ampliación, se procede al análisis de 80 empresas, de las cuales se dispone de datos de empleo de 75 de ellas, con un total de 4.123 de empleos, encontrándose entre ellos 2.627 correspondientes a óptica oftálmica, estimados para 2013. La estructura empresarial, de empleo, de facturación y gastos de personal se observan en la Figura 2. 
Figura 2

Composición del sector considerando empresas fabricantes e importadoras

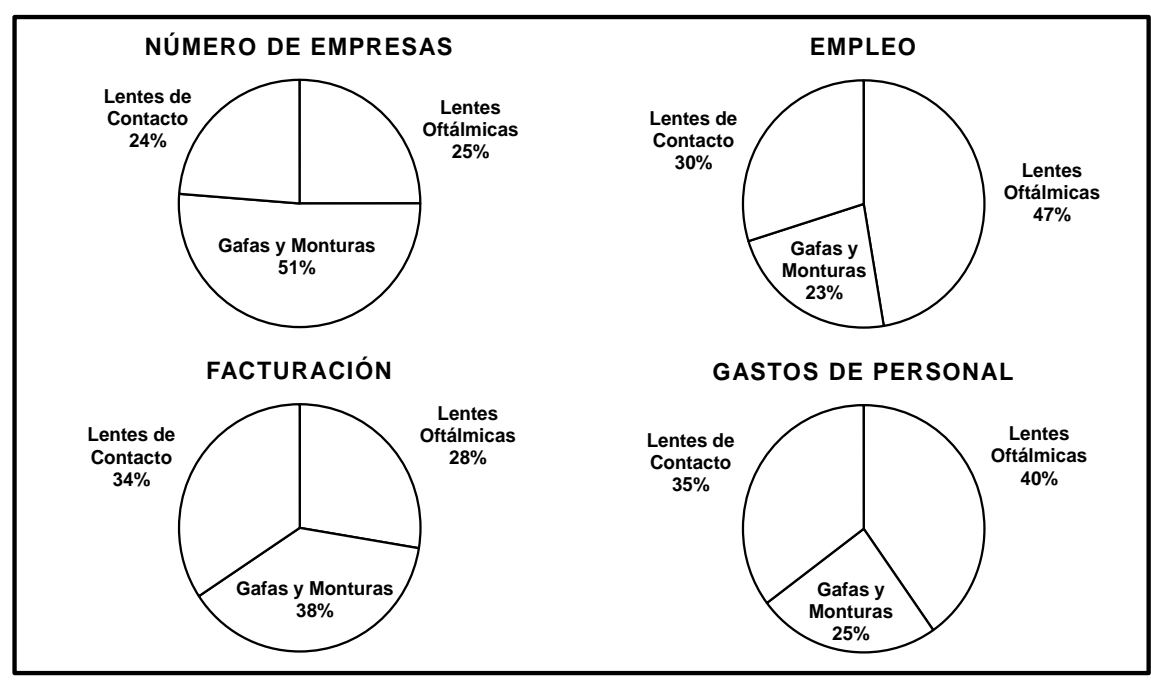

Fuente: Elaboración propia. Sabi (2015).

Los datos sobre la estructura sectorial los encontramos en la Tabla . De ella, se recoge el tamaño del sector, que alcanza 707 millones de euros de facturación en 2013. De dicha cantidad un 49\% corresponde a producción nacional, mientras que el resto proviene de la distribución de productos importados. Siendo el nivel de importaciones de estas empresas seis veces superior a las exportaciones, y el valor añadido promedio de las tres actividades es del 17\% de la facturación total. A pesar de que la mayoría de las empresas de monturas son distribuidoras, la mayor parte de la producción se lleva a cabo por empresas de monturas y gafas. El nivel de exportaciones e importaciones de este tipo de empresas especializadas que aparece en la Tabla 5 corresponde a aquellas de las empresas. Dichos valores no coinciden con las exportaciones e importaciones de productos de óptica oftálmica que se realiza en España, que está protagonizado por el segmento más concreto de gafas de sol (Barrera, Mainar, \& Vallés, 2016; Barrera, 2016), sino que son las exportaciones e importaciones propias de las empresas proveedoras de productos de la óptica.

La principal diferencia que se encuentra al considerar las empresas importadoras es el incremento de la participación de gafas y monturas, especialmente en el número de empresas y en la facturación. Esta actividad se diferencia especialmente de las otras dos por el valor añadido derivado del empleo, de un 10\% de la facturación, en contraste con el 25\% de lentes oftálmicas y el $17 \%$ de lentes de contacto. 
Tabla 5

Estructura productiva del sector de fabricantes e importadores de óptica oftálmica en España

\begin{tabular}{|c|c|c|c|}
\hline & Lentes Oftálmicas & Gafas y Monturas & Lentes de Contacto \\
\hline Número de empresas & 20 & 41 & 19 \\
\hline Empleo & 1242 & 598 & 783 \\
\hline Producción & 45267 & 221786 & 77325 \\
\hline Exportación & 13451 & 29576 & 20503 \\
\hline Importación & 163581 & 77045 & 185830 \\
\hline Facturación & 195397 & 269255 & 242652 \\
\hline Gastos de personal & 48420 & 29510 & 42375 \\
\hline
\end{tabular}

Fuente: Elaboración propia. Sabi (2015). Datos en miles de euros.

La mitad de las empresas de óptica oftálmica están especializadas en las monturas de gafas y gafas de sol, Tabla 6, un $80 \%$ de éstas tienen menos de 20 empleados. El 50\% de las empresas de lentes oftálmicas y el 32\% de las de lentes de contacto tienen menos de 20 trabajadores, mientras que el grupo de ambas abarca el 90\% del total de empresas de más de 100 empleados. De lo que se deduce, al igual que para el conjunto de empresas fabricantes previamente analizado, que las empresas de lentes son aquellas que alcanzan una escala de funcionamiento más alta. En este caso se observa la ausencia de empresas entre 50 y 100 empleados de lentes oftálmicas y monturas.

Tabla 6

Tamaño de las empresas fabricantes e importadoras de óptica oftálmica

\begin{tabular}{|c|c|c|c|c|}
\hline & $\begin{array}{c}\text { Menos de 20 } \\
\text { empleados }\end{array}$ & $\begin{array}{c}\mathbf{2 0 - 5 0} \\
\text { empleados }\end{array}$ & $\begin{array}{c}\mathbf{5 0 - 1 0 0} \\
\text { empleados }\end{array}$ & $\begin{array}{c}\text { Más de 100 } \\
\text { empleados }\end{array}$ \\
\hline Lentes oftálmicas & 10 & 6 & 0 & 4 \\
\hline Monturas y gafas & 33 & 7 & 0 & 1 \\
\hline Lentes de contacto & 6 & 5 & 3 & 5 \\
\hline
\end{tabular}

Fuente: Elaboración propia. Sabi (2014). Datos en número.

La localización geográfica está muy concentrada en Madrid y Cataluña, donde se encuentran distribuidas el $40 \%$ de empresas en cada una de estas comunidades, como se observa en la Figura 3, destacando las empresas de monturas en Cataluña mientras que en la Comunidad de Madrid lo hacen las de lentes de contacto. El número de empresas de lentes oftálmicas es superior en la Comunidad de Madrid, con el $28 \%$ del total, mientras que Cataluña recoge el $18 \%$. 
Figura 3

Localización geográfica del sector de la óptica oftálmica en España

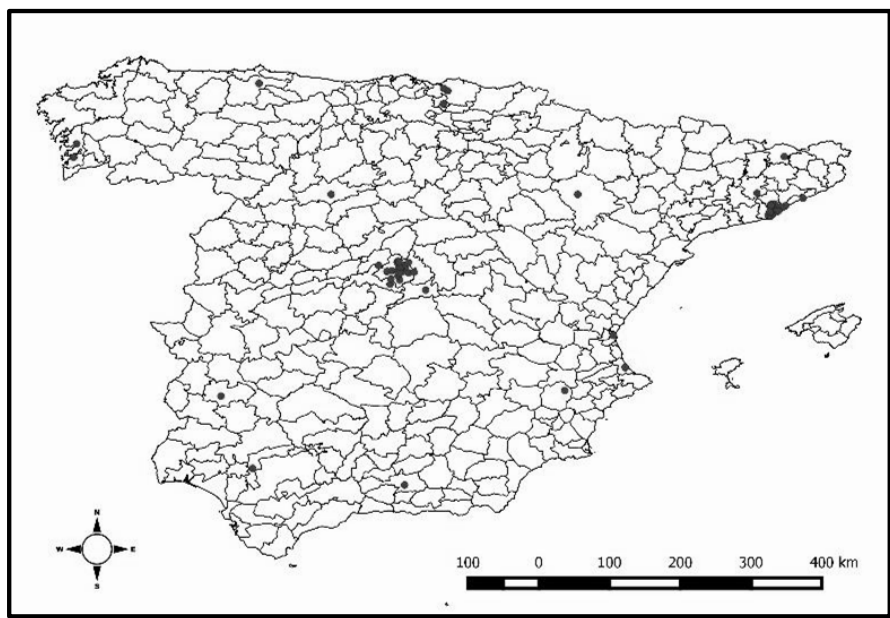

Fuente: Elaboración propia. Sabi (2015).

\section{EVOLUCIÓN RECIENTE DEL SECTOR}

Con objeto de conocer la evolución del sector durante los años de crisis y obtener una primera aproximación de la capacidad de respuesta del mismo ante los efectos coyunturales, se procede a analizar los datos correspondientes al periodo comprendido entre 2008 y 2014. En primer lugar se analizan los datos de producción según la Encuesta Anual Industrial de Productos (EIP) (INE, 2017) y posteriormente se profundizará a nivel empresarial a través de los datos obtenidos de su información contable.

La principal ventaja de la información recogida de la EIP es el detalle de productos que ofrece, que diferencia entre los siguientes: lentes de contacto; lentes para gafa: completamente trabajadas en las dos caras, no correctoras, correctoras monofocales, las demás correctoras, otras; y gafas: de sol, correctoras protectoras y otras, monturas de gafas o de artículos similares de plástico y de otros materiales. El alto nivel de detalle es al mismo tiempo una desventaja debido a la desinformación de la base de datos a este nivel, derivado del número de empresas que componen el sector. Como resultado, la EIP aporta datos de los siguientes productos: lentes de contacto, lentes correctoras monofocales, lentes correctoras no monofocales y gafas correctoras, protectoras y otras, en términos de valor, quedando desierta la información sobre gafas de sol y monturas, mientras que en términos de cantidades los datos recogen los datos de lentes de contacto y lentes oftálmicas.

La producción en términos de valor, Tabla 7, ha disminuido en el periodo de 2008 a 2014 en un 24\%. Esta disminución ha sido más pronunciada en el caso de 
lentes monofocales, cuya producción ha disminuido en un 39\%. Contrastando con el comportamiento de lentes de contacto, que mejora un $68 \%$ pasando de suponer el $6 \%$ del total al $13 \%$. Durante todo el periodo, las lentes multifocales son las protagonistas de la producción, manteniéndose cercana al 70\% del total.

Tabla 7

Evolución de la producción de óptica oftálmica en España (I)

\begin{tabular}{|c|r|r|r|r|r|r|r|r|r|}
\hline & $\mathbf{2 0 0 8}$ & $\mathbf{2 0 0 9}$ & $\mathbf{2 0 1 0}$ & $\mathbf{2 0 1 1}$ & $\mathbf{2 0 1 2}$ & $\mathbf{2 0 1 3}$ & $\mathbf{2 0 1 4}$ & \multicolumn{1}{|c|}{$\mathbf{2 0 1 5}$} & $\mathbf{2 0 1 6}$ \\
\hline LC & 10038 & 11584 & 14901 & 16101 & 16767 & 16636 & 16832 & 17030 & 18702 \\
\hline LOM & 38359 & 37754 & 39944 & 37813 & 33244 & 29519 & 23494 & 37602 & 38398 \\
\hline LOMM & 118667 & 111542 & 100320 & 98387 & 92319 & 90406 & 87832 & 103510 & 107768 \\
\hline LCP & 5143 & 4209 & 3585 & 4345 & 4450 & 4057 & 3484 & 4240 & 4351 \\
\hline Total & 172207 & 165089 & 158750 & 156646 & 146780 & 140618 & 131642 & 162382 & 169219 \\
\hline
\end{tabular}

Nota: Datos en miles de euros. LC: Lentes de contacto; LOM: Lentes oftálmicas monofocales; LOMM: Lentes oftálmicas multifocales; LCP: Lentes correctoras y protectoras. Los datos de producción de monturas no están disponibles por secreto estadístico.

Fuente: Elaboración propia. EIP (INE, 2017).

El comportamiento del valor de la producción contrasta con aquel de cantidad producida, Tabla 8, con un valor en 2014 un 25\% superior al de 2008. La principal causa de esta diferencia radica en la cantidad producida de lentes de contacto, que en 2014 fue 7 veces superior a la de 2008, siendo la cantidad producida de lentes oftálmicas monofocales un $25 \%$ superior, mientras que la de lentes oftálmicas progresivas ha disminuido en un $37 \%$. Un factor determinante en esta diferencia es la vida media del producto, siendo inferior en lentes de contacto por una mayor frecuencia en su reemplazo, cuando se tienen en cuenta las lentes de contacto desechables. La diferencia en la vida media de lentes de contacto mensuales y gafas completas, y sus efectos sobre la rentabilidad en la venta al consumidor final, se analiza en Ibáñez (2009).

$\mathrm{El}$ incremento en la cantidad de lentes de contacto, notablemente superior a su valor monetario, coincide en el tiempo con el desarrollo de nuevos materiales y diseños de lentes de contacto especialmente en aquellos utilizados en lentes desechables, lo que concuerda con el aumento sucedido, posiblemente derivado del incremento en el reemplazo por parte del usuario final ${ }^{5}$. Por otra parte la

\footnotetext{
${ }^{5}$ El incremento de producción de lentes de contacto desechables se da al tiempo que se estanca y disminuye el uso de lentes convencionales para uso habitual, como se deriva del estudio realizado por Santodomingo et al. (2017). Sin embargo, (1) la mejora de los materiales y las geometrías de lentes de contacto no deja claro que la estandarización de las lentes conlleve una mayor intolerancia por parte de usuario de éstas y (2) existe una tendencia creciente a utilizar lentes personalizadas para el tratamiento de anomalías corneales, Santodomingo et al. (2009) analizan este tipo de tratamiento. Además, los motivos principales que conllevan al abandono del uso de lentes de contacto, según Ibáñez (2012), son la cirugía refractiva $(22,3 \%)$ y la incomodidad (23,2\%), entre otros, como los indicados por Nichols et al. (2013): la utilización de productos de mantenimiento. Los nuevos materiales de lentes desechables buscan precisamente el incremento
} 
diferencia entre valor y cantidades en el caso de lentes monofocales se deriva de la disminución en el precio unitario del producto, expuesto a una fuerte competencia internacional. Por su lado, la producción de lentes oftálmicas progresivas ha disminuido más en cantidad que en valor, lo que puede deberse al desarrollo de nuevos diseños y el incremento de su valor añadido, que hayan contrarrestado, en parte, la disminución de la demanda con una mejora de la calidad del producto, manteniéndose más localizada en España este tipo de producción, como ya señala Barrera et al. (2016), relacionado con el análisis de relocalización realizado por Martínez y Merino (2017), donde se indica que la producción que menos tiende a deslocalizarse es aquella de mayor calidad o complejidad.

\section{Tabla 8}

Evolución de la producción de óptica oftálmica en España (II)

\begin{tabular}{|c|c|c|c|c|c|c|c|c|c|}
\hline & $\mathbf{2 0 0 8}$ & $\mathbf{2 0 0 9}$ & $\mathbf{2 0 1 0}$ & $\mathbf{2 0 1 1}$ & $\mathbf{2 0 1 2}$ & $\mathbf{2 0 1 3}$ & $\mathbf{2 0 1 4}$ & $\mathbf{2 0 1 5}$ & $\mathbf{2 0 1 6}$ \\
\hline LC & 491 & 834 & 1824 & 2761 & 3721 & 3816 & 3817 & 4005 & 4187 \\
\hline LCM & 4134 & 3809 & 5665 & 5319 & 5136 & 5090 & 5092 & 7463 & 5927 \\
\hline LCMM & 5082 & 4379 & 3779 & 3548 & 3301 & 2711 & 3214 & 3771 & 4038 \\
\hline Total & 9707 & 9021 & 11268 & 11627 & 12159 & 11616 & 12123 & 15239 & 14152 \\
\hline
\end{tabular}

Nota: Datos en miles de unidades. LC: Lentes de contacto; LOM: Lentes oftálmicas monofocales; LOMM: Lentes oftálmicas multifocales. Los datos de producción de monturas no están disponibles por secreto estadístico.

Fuente: Elaboración propia. EIP (INE, 2017).

La alta representación de las empresas de monturas y gafas sobre el total de empresas de óptica hace necesario profundizar en estos datos a nivel empresarial, por la insuficiente información disponible en estadísticas oficiales, que si bien ofrecen una alta fiabilidad (Martín-Guzmán, 2016) aún presentan limitación, como por ejemplo el secreto estadístico. El uso de la contabilidad de las empresas para el análisis de la evolución del sector, permite conocer el comportamiento en todas las líneas de actividad, por lo que se procede a hacer uso de la información obtenida de Sabi para así poder profundizar en la información que no está disponible a través de estadísticas oficiales. Debido a la falta de datos para todos los periodos se disminuye el número de empresas analizadas a 50, de las 80 utilizadas en el análisis de la estructura sectorial y se decide realizarlo para el periodo entre 2008 y 2013 debido a que el número de empresas disponibles en 2014, en el momento del análisis, es aún menor. A partir de las

de la comodidad a través de una menor perdida de humectación y una mayor transmisibilidad al oxígeno. Una adaptación personalizada sigue siendo posible con una especialización mayor del profesional óptico-optometrista, una mejora de los equipos de adaptación (topógrafos), y un incremento en el precio final de la lente de contacto. En cualquier caso, la determinación de si el incremento en la estandarización de los parámetros de lentes de contacto es o no causante de un incremento en el abandono de lentes de contacto, no es objeto de este estudio. 
empresas de las que se dispone de datos para todos los periodos, se considera la ponderación sobre los valores analizados anteriormente para 2013, obteniendo los datos reflejados en la Tabla 9. Se asume la limitación derivada de: considerar que no se han creado ni destruido empresas en el periodo y que la participación de las empresas disponibles sobre el total ha sido la existente en 2013, siendo la manera disponible para observar la imagen cronológica en los años de crisis.

Tabla 9

Evolución de la facturación del sector de la óptica oftálmica por actividad

\begin{tabular}{|c|c|c|c|c|c|c|}
\hline & $\mathbf{2 0 0 8}$ & $\mathbf{2 0 0 9}$ & $\mathbf{2 0 1 0}$ & $\mathbf{2 0 1 1}$ & $\mathbf{2 0 1 2}$ & $\mathbf{2 0 1 3}$ \\
\hline Lentes oftálmicas & 291701 & 226235 & 239422 & 231175 & 216847 & 195397 \\
\hline Monturas y gafas & 300570 & 237786 & 264169 & 274094 & 257725 & 269255 \\
\hline Lentes de contacto & 198866 & 205670 & 226615 & 235206 & 236669 & 243489 \\
\hline
\end{tabular}

Nota: Datos en miles de euros.

Fuente: Elaboración propia. Sabi (2015).

La evolución ha sido diferente en cada una de las actividades consideradas, siendo la tendencia de lentes oftálmicas negativa, con una pérdida del 33\% desde 2008. La actividad de gafas y monturas ha disminuido en un $10 \%$ desde 2008. Sin embargo, si se considera la variación desde 2009 ha mejorado su facturación en un 13\%. La actividad de lentes de contacto muestra tendencia positiva en todo el periodo, con una mejora del 22\%. Contrastando con los datos obtenidos del INE entre 2008 y 2013, se observa que las expectativas de la información contable son más negativas: la disminución de lentes oftálmicas derivada de su contabilidad es del 33\%, mientras que la de producción obtenida de la EIP es del 23\%; y el incremento de lentes de contacto registrado por la contabilidad es del $22 \%$, frente al crecimiento del $66 \%$ marcado en su producción. De ello se concluye que el comportamiento de los ingresos por ventas de las empresas de óptica oftálmica ha sido más negativo que el de su fabricación, no siendo posible considerar el caso de gafas y monturas por disponer solo de información parcial a través de la EIP.

En términos totales, los datos a nivel sectorial muestran un $10 \%$ de decrecimiento, explicado por el comportamiento de las lentes oftálmicas, habiéndose incrementado un 9\% la participación de la actividad de lentes de contacto entre 2008 y 2013, que es el rango de años para los que se dispone de un mayor número de empresas.

Otro factor destacado es la merma del 15\%, sufrida entre 2008 y 2009, y la mejora en términos totales de 2009 a 2013, siendo las lentes oftálmicas la única actividad que evoluciona negativamente. El comportamiento de la actividad de lentes de contacto ha sido más positivo que el del conjunto empresarial al que pertenece. Influido, como ya se ha indicado en el análisis de producción, por 
nuevos materiales y diseños de lentes de contacto, que pueden haber sido responsables del mantenimiento y mejora de la tendencia de éstas, pero también puede haberse causado por una situación de partida más negativa, habiendo incrementado su peso en el total en un 36\%. Sin embargo, este incremento parece venir derivado de reposiciones de nuevos clientes y no del incremento del mercado, lo que indica la madurez del mercado, según Santodomingo et al. (2017)

La actividad de gafas y monturas sufrió una recesión del 21\% de 2008 a 2009, mientras que desde 2009 a 2013 ha incrementado sus ingresos en un 13\%, lo que contrasta con la disminución del $17 \%$ sufrida por la producción de las gafas correctoras a las que hace referencia la EIP. Esta diferencia, además de deberse a la diferencia en la inclusión de productos, también se debe al incremento de monturas y gafas importadas y exportadas en el periodo, y que no recoge la EIP. Por una parte el incremento de las importaciones puede haber sustituido la producción nacional consumida a nivel doméstico, pero el incremento de las exportaciones de producto extranjero también puede haber influido positivamente la cifra de negocios de las empresas de monturas y gafas.

Los últimos años se ha producido un importante cambio en la forma de comercialización en la economía. En concreto, en la economía española el número de empresas que han realizado ventas por internet se ha visto incrementado en un 84\% entre 2008 y 2016, según la Encuesta de uso de TIC y Comercio Electrónico (CE) en las empresas (INE, 2017). Habiéndose producido un cambio más destacable en las empresas de menor tamaño (un $88 \%$ en las empresas de 10 a 49 empleados, frente a un $42 \%$ en empresas de 250 o más empleados), tal y como se deriva de la Tabla 10. Sin embargo, la variación absoluta más alta se ha producido entre las empresas más grandes.

Tabla 10

Empresas que han realizado ventas por comercio electrónico

\begin{tabular}{|c|c|c|c|c|c|c|c|c|c|}
\hline Periodo & $\mathbf{2 0 0 8}$ & $\mathbf{2 0 0 9}$ & $\mathbf{2 0 1 0}$ & $\mathbf{2 0 1 1}$ & $\mathbf{2 0 1 2}$ & $\mathbf{2 0 1 3}$ & $\mathbf{2 0 1 4}$ & $\mathbf{2 0 1 5}$ & $\mathbf{2 0 1 6}$ \\
\hline Total & 11 & 13 & 12 & 14 & 14 & 18 & 18 & 20 & 20 \\
\hline De 10 a 49 & 10 & 12 & 11 & 12 & 12 & 16 & 15 & 18 & 18 \\
\hline De 50 a 249 & 18 & 19 & 19 & 24 & 25 & 27 & 28 & 29 & 30 \\
\hline De 250 y más & 29 & 30 & 32 & 34 & 34 & 37 & 39 & 40 & 41 \\
\hline
\end{tabular}

Nota: Datos en \%.

Fuente: Elaboración propia. INE (2017).

Las empresas industriales presentan una evolución distinta a la del conjunto de la economía. En este caso las empresas que más han incrementado sus ventas por internet son las de tamaño medio, con un aumento del $67 \%$ y un cambio absoluto también más alto. De manera similar, pero más diferenciada, el colectivo de empresas dentro del que encontramos a las empresas de óptica 
(CNAE 26-33) ha incrementado sus ventas por internet en un 43\%. Sin embargo el porcentaje sólo de las de 50 a 249 trabajadores ha sido del 70\% (Tabla 11 y Tabla 12). Esta información indica la gran diferencia en estrategias de venta que se encuentra entre los diferentes tamaños empresariales.

Tabla 11

Empresas industriales que han realizado ventas por comercio electrónico (CNAE-10-39)

\begin{tabular}{|c|c|c|c|c|c|c|c|c|c|}
\hline Periodo & $\mathbf{2 0 0 8}$ & $\mathbf{2 0 0 9}$ & $\mathbf{2 0 1 0}$ & $\mathbf{2 0 1 1}$ & $\mathbf{2 0 1 2}$ & $\mathbf{2 0 1 3}$ & $\mathbf{2 0 1 4}$ & $\mathbf{2 0 1 5}$ & $\mathbf{2 0 1 6}$ \\
\hline Total & 12 & 14 & 12 & 13 & 14 & 17 & 16 & 19 & 20 \\
\hline De 10 a 49 & 10 & 12 & 9 & 10 & 11 & 14 & 12 & 15 & 16 \\
\hline De 50 a 249 & 19 & 22 & 22 & 24 & 27 & 30 & 29 & 31 & 32 \\
\hline De 250 y más & 43 & 40 & 43 & 46 & 48 & 46 & 52 & 50 & 51 \\
\hline
\end{tabular}

Nota: Datos en \%.

Fuente: Elaboración propia. INE (2017).

Tabla 12

Empresas correspondiente a: Productos informáticos, electrónico y ópticos, material y equipo eléctrico y similares (CNAE 26-33), que han realizado ventas por comercio electrónico

\begin{tabular}{|c|c|c|c|c|c|c|c|c|c|}
\hline Periodo & $\mathbf{2 0 0 8}$ & $\mathbf{2 0 0 9}$ & $\mathbf{2 0 1 0}$ & $\mathbf{2 0 1 1}$ & $\mathbf{2 0 1 2}$ & $\mathbf{2 0 1 3}$ & $\mathbf{2 0 1 4}$ & $\mathbf{2 0 1 5}$ & $\mathbf{2 0 1 6}$ \\
\hline Total & 10 & 12 & 11 & 10 & 10 & 12 & 13 & 15 & 15 \\
\hline De 10 a 49 & 8 & 10 & 8 & 7 & 6 & 8 & 9 & 11 & 11 \\
\hline De 50 a 249 & 15 & 20 & 22 & 21 & 21 & 25 & 22 & 25 & 25 \\
\hline De 250 y más & 46 & 41 & 50 & 49 & 48 & 47 & 55 & 51 & 57 \\
\hline
\end{tabular}

Nota: Datos en \%.

Fuente: Elaboración propia. INE (2017).

La mayoría de las empresas de óptica son pequeñas empresas, aunque existe un pequeño número de empresas más grandes que concentran la mayor parte del mercado. Son precisamente estos dos segmentos de tamaño empresarial los que menos variación han sufrido en sus ventas por comercio electrónico ${ }^{6}$. También es interesante la evolución de este tipo de comercio en los años posteriores a la crisis, en que las empresas más pequeñas disminuyeron el porcentaje de ventas de este tipo, influido por una menor capacidad para competir vía precios y tiempos de envío.

Las empresas de óptica presentan diferentes capacidades de diferenciación en función del tipo de producto: las lentes oftálmicas pueden diferenciarse en precio, pero sólo en aquellos tipos de lentes que no requieren un tipo de fabricación personalizado. Gran parte de las lentes oftálmicas monofocales se importan de

\footnotetext{
${ }^{6}$ Dentro de las de Productos informáticos, electrónicos y ópticos material y equipo eléctrico maquinaria y equipo mecánico, vehículos a motor, material de transporte, muebles, reparación, maquinaria y equipo.
} 
países en los que los costes laborales son más bajos que en España, esta deslocalización es menos factible para lentes progresivas y de fabricación exclusiva. De manera que una forma de diferenciación es la creación de lentes personalizadas para todos los tipos de lentes, incrementando la calidad de estas lentes que sí serán fabricadas en España y se ofrecerán con un precio final más alto. Asociado a este tipo de diferenciación encontramos la inclusión de la marca en las lentes, a través del labrado de símbolos específicos para las marcas que ofrecen estos niveles de calidad más altos.

Asociado a ello es interesante hacer referencia a la estrategia de Essilor, uno de los principales proveedores de lentes oftálmicas a nivel mundial, que se resumen en "innovación, cercanía y compromiso", basando su estrategia en la innovación, el acercamiento al cliente final y acciones de concienciación social (Almena, 2017). El acercamiento a su cliente más próximo (establecimientos de óptica), se basa en la oferta de productos más valorados por los especialistas en la adaptación de lentes (ópticos-optometristas) que deben garantizar una mejor calidad visual. Este tipo de estrategia es valorada por los ópticos-optometristas, que son los que deben de convencer al cliente final de cuál es la mejor opción para la visión. El acercamiento al cliente final es por tanto crucial, siendo precisamente uno de los más complicados de implementar en el comercio electrónico, que favorece sobre todo el liderazgo en precios o la imagen de marca.

Por otro lado, dentro del segmento de monturas existe una amplia gama de calidades. Las de calidad más baja pueden permitirse competir en precios, provenientes de los países del este asiático. Sin embargo, las de calidades más altas provienen en su mayoría de fabricación italiana, entre éstas, la diferenciación predominante es la imagen de marca. Un aspecto cada vez más influyente en este segmento es el de la utilización de marketing en redes sociales, como es el caso de Hawkers. Esta empresa, en tan sólo unos años, ha conseguido convertirse en una de las marcas de monturas más populares (Hawkers revoluciona la industria de gafas de sol, 2015). Este tipo de marketing comenzó en un principio asociada a gafas de bajo precio y con un diseño que inspirado en patentes obsoletas de marcas como Ray Ban, haciendo posible alcanzar una buena posición en el mercado durante el periodo de crisis. Una vez instauradas en el mercado, se dispusieron a incrementar los canales de venta, de venta online a venta en establecimientos físicos de óptica y también establecimientos propios.

Las estrategias desarrolladas por las empresas de lentes de contacto son más similares a las que utilizan las de lentes oftálmicas, diferenciándose en calidad y diseños más cómodos. Cada vez más, se facilitan las adaptaciones por parte de sus clientes (los ópticos-optometristas), reduciendo el número de parámetros y flexibilizando la cobertura de estos parámetros. La ampliación de tipos de diseños de lentes de contacto ha permitido también ampliar el nicho de mercado: geometrías inversas para el control de la miopía, la corrección de la visión en 
casos de corneas irregulares, o la adaptación en los más jóvenes gracias a materiales con una mayor permeabilidad al oxígeno. Destaca, también en este segmento, el marketing digital.

\section{LA PRODUCCIÓN DE ÓPTICA EN EUROPA}

Según las estadísticas de Producción vendida, exportaciones e importaciones de Eurostat (2017) el valor de la fabricación de óptica oftálmica en Europa ${ }^{7}$ en 2014 fue de 10.540 millones de euros, aproximadamente un 0,1\% del total del PIB europeo. Este primer dato a nivel europeo permite comparar la posición de España, que representa el 0,01\% del PIB español, y que coincide con el valor promedio. Los países en que la fabricación de óptica oftálmica tiene una mayor importancia en términos de PIB son Italia, Alemania y Portugal, mientras que en términos absolutos son Italia, Alemania y Reino Unido.

Tabla 13

Evolución de la producción de óptica oftálmica en Europa (1995-2014)

\begin{tabular}{|c|c|c|c|c|c|c|c|c|c|c|c|}
\hline & $\mathbf{1 9 9 5}$ & $\mathbf{1 9 9 6}$ & $\mathbf{1 9 9 7}$ & $\mathbf{1 9 9 8}$ & $\mathbf{1 9 9 9}$ & $\mathbf{2 0 0 0}$ & $\mathbf{2 0 0 1}$ & $\mathbf{2 0 0 2}$ & $\mathbf{2 0 0 3}$ & $\mathbf{2 0 0 4}$ & $\mathbf{2 0 0 5}$ \\
\hline Francia & 721 & 731 & 777 & 771 & 704 & 665 & 716 & 700 & 687 & 714 & 696 \\
\hline Alemania & 683 & 802 & 721 & 743 & 802 & 779 & 854 & 925 & 1214 & 916 & 949 \\
\hline Italia & 836 & 1340 & 1538 & 1634 & 1320 & 1499 & 1930 & 1912 & 1974 & 1683 & 1796 \\
\hline España & 153 & 152 & 163 & 194 & 174 & 167 & 231 & 227 & 244 & 206 & 207 \\
\hline Reino Unido & 321 & 385 & 494 & 524 & 579 & 639 & 711 & 688 & 594 & 725 & 743 \\
\hline
\end{tabular}

Nota: Datos en millones de euros.

Fuente: Elaboración propia. Eurostat (2017).

Las estadísticas de producción de bienes manufacturados de Eurostat, facilitan información por grupos Prodcom, recogiendo información de los mismos productos que la EIP $^{8}$. El problema de desinformación que se observaba con anterioridad sigue existiendo para el caso de España, no así para los países cuyos sectores de óptica oftálmica alcanzan un mayor tamaño, siendo posible extraer la evolución de los países más representativos, como se indica en la Tabla 13. Es especialmente destacable la evolución de la industria óptica italiana, que hasta 2008 había crecido un 300\%, industria que ha basado su crecimiento en el

\footnotetext{
${ }^{7}$ Países con cobertura de datos en Prodcom: Francia, Países Bajos, Alemania, Italia, Reino Unido, Dinamarca, Grecia, Portugal, España, Finlandia, Polonia, Hungría, Rumanía, Eslovenia y Croacia.

${ }^{8}$ Es la utilizada en la primera parte del Apartado 4.
} 
desarrollo local a través de la adquisición de tecnología foránea (Belussi, 2015) y la británica, con un $130 \%$, siendo ambas las que muestran mayor crecimiento para el periodo de 1995 a 2014. Sin embargo la única economía que no se ha visto marcadamente castigada en los años de crisis ha sido Alemania. En España alcanzó un crecimiento del $42 \%$ hasta el comienzo de la crisis, decreciendo un 25\% hasta 2014, mientras que en el periodo entre 2014 y 2016 es la que más se ha incrementado, un $28 \%$.

Los diferentes países analizados presentan una especialización singular en cada uno de los casos. Los principales productores de lentes oftálmicas son Alemania y Francia, con un valor de 600 y 400 millones de euros cada una en 2014. El principal productor de lentes de contacto ${ }^{9}$ es Reino Unido que fabricó 200 millones de euros en 2014. El principal productor de monturas y gafas de sol es Italia, que en 2014 produjo 620 y 770 millones de euros. Con la limitación derivada del secreto estadístico en el caso de producción, España destacaría por su producción en lentes oftálmicas, aunque dentro de los cinco países principales se sitúa en último lugar.

La reacción que los diferentes productos han demostrado a la coyuntura económica se observa en la producción de óptica oftálmica en cada uno de los países, observándose una mayor versatilidad de monturas y gafas, especialmente en el caso de Italia, y un crecimiento más sostenido de la producción relacionada con lentes oftálmicas y de contacto, como es el caso de Alemania y Reino Unido. Es posible que ello también radique en el tipo de material del producto, ya que, por ejemplo, España se caracterizó por el suministro de lentes minerales y monturas metálicas, productos menos innovadores que las lentes oftálmicas orgánicas y de pasta. El caso de Francia muestra una estabilidad muy marcada en el largo plazo, que se ve favorecida en el comienzo de la crisis, cuyos efectos no sufre hasta 2011, posiblemente limitado por la desinformación de los datos sobre fabricación de monturas e influido por procesos de deslocalización productiva de lentes oftálmicas hacia otros países, lo que se deduce de un incremento de las importaciones del 22\% entre 2009 y 2013 en cantidad y del 16\% en valor.

\section{CONCLUSIONES}

La estructura de la óptica oftálmica en la economía española se caracteriza por el alto valor añadido que aporta derivado del empleo. Sin embargo, el comportamiento por productos muestra singularidades, siendo especialmente alto el de lentes oftálmicas y lentes de contacto, no así el segmento de monturas y gafas. Este último se caracteriza por una atomización mayor de la estructura productiva, que no parece coincidir con la fabricación industrial, sino con empresas importadoras y distribuidoras de gafas y monturas. La tendencia

\footnotetext{
${ }^{9}$ Sin incluir a Irlanda, cuyos datos se encuentran bajo secreto estadístico.
} 
creciente de creación de este último tipo de empresas hace necesario considerarlas como parte del sector por ser aquellas más representativas dentro del número total de empresas.

La evolución que ha tenido la cifra de ventas del sector durante los años de crisis demuestra una alta versatilidad a los efectos coyunturales, sufriendo una bajada del 25\% entre 2008 y 2014 en términos de producción y del 10\% en términos de facturación del sector. Los daños sufridos en la producción están explicados por los derivados de lentes oftálmicas que han sido de un $40 \%$ para la producción de monofocales y del $25 \%$ para multifocales, siendo el perjuicio en las ventas del 33\%. Las monturas también han sufrido los efectos de la crisis en un $10 \%$. Sin embargo, tanto la producción como las ventas de lentes de contacto han mostrado una tendencia positiva durante todo el periodo de ralentización económica, siendo más positivo el incremento en producción que el de ventas.

El comportamiento de lentes de contacto tiene dos justificaciones, por una parte se han producido mejoras de producto tanto en materiales como en diseño, lo que ha contrarrestado la tendencia general de la demanda. Estas mejoras se han producido en todo tipo de lentes de contacto, pero han influido especialmente en aquellas de reemplazo frecuente, lo que ha provocado que el incremento de producción en cantidades haya sido mucho más superior que el incremento en valor. Esto ha dado lugar a una modificación en la composición del sector, que ha pasado de un reparto desfavorable en lentes de contacto, con un $25 \%$ en 2008 a un reparto más equitativo en el cual las lentes de contacto incluso superan la participación de lentes oftálmicas.

La diferencia entre la pérdida en lentes monofocales y multifocales también se relaciona con las variaciones en cantidades. Las primeras, las monofocales, muestran una tendencia hacia la disminución en precios, mientras que las segundas, muestran haber mejorado el valor añadido unitario del producto. El comportamiento de las multifocales, de manera similar a las lentes de contacto, se ha visto afectado por procesos de innovación y mejora de diseños de lentes, más destacables que aquellos sucedidos en lentes monofocales.

La gran especialización de la materia de estudio tiene la limitación de no poder determinar los niveles de producción en aquellos productos fabricados por un número pequeño de empresas, lo que se aborda analizando en detalle la contabilidad de las empresas del sector, de donde se deduce que las monturas y gafas representan una parte muy importante de la producción y la facturación del sector, que se ha visto fuertemente afectada en el comienzo de la crisis, pero que ha respondido positivamente en el periodo entre 2009 y 2013, con un crecimiento del $13 \%$.

Teniendo en cuenta la evolución de otras economías, se observa que aquellos países especializados en lentes han sido los que menos han sufrido los efectos 
de la coyuntura económica, lo que hace especialmente atractivos este tipo de productos. Por esta razón, a pesar de observarse una cierta tendencia hacia la especialización de la venta de monturas y gafas, apostar por el fortalecimiento de la investigación y desarrollo de lentes parece ser un factor clave en el crecimiento del sector. Esto no descarta apostar por incentivos semejantes en la actividad de monturas y gafas, a pesar de que las lentes muestran un valor añadido de mayor cuantía y más beneficioso para la dinamización del resto de la economía.

A nivel microeconómico es importante prestar atención a las estrategias de diferenciación que han implementados las empresas que más han crecido en los últimos años. Entre éstas, destaca la diferenciación a través del uso de imagen de marca y las estrategias intensivas en marketing digital. Sin embargo, es importante tener en cuenta que la tendencia generalizada del sector es incrementar el nivel de comercio electrónico a una menor velocidad que el resto de la economía, especialmente en las empresas más pequeñas y más grandes. Esta situación se da, muy probablemente, por las ventajas que aportan la cercanía al cliente, y que no está patente en el comercio electrónico. Por ello, se requieren acciones complementarias de fidelización on-line, que contrarresten las dificultades para diferenciarse en costes.

\section{REFERENCIAS BIBLIOGRÁFICAS}

AGENCIA TRIBUTARIA. (2015). Tabla del impuesto sobre actividades económicas. Recuperado el 01 de 04 de 2015, de Agencia Tributaria: https://www2.agenciatributaria. gob.es/ADUA/internet/es/aeat/dit/adu/adws/certificados/Tabla_de_epigrafes_IAE.pdf

ALMENA, F. (2017). 2017, un gran año para Essilor. Optimoda.

BARRERA, M. (2016). "International trade between Spain-Morocco-Portugal: Are there any opportunities for optical products". Atlantic Review of Economics, I/(1), pp. 1-23.

BARRERA, M., CAUSAPÉ, A., \& VALLÉS, J. (2015). "Dissagregation of sectors in Social Accounting Matrices using a customized Wolsky method". Applied Economics Letters, 22, pp. 1020-1024.

BARRERA, M., MAINAR, A., \& VALLÉS, J. (2016). "Ophthalmic-optical foreign sector". Regional and Sectoral Economic Studies, 16(1), pp. 45-55.

BELUSSI, F. (2015). "The international resilence of Italian industrial districts/clusters (ID/C) between knowledge re-shoring and manufacturing off (near)-shoring". Journal of Regional Research(32), pp. 89-113.

CEGARRA NAVARRO, J. G., \& RODRIGO MOYA, B. (2003). "Orientadores del aprendizaje relacional". Cuadernos de Administración, 16(26), pp. 79-97.

CEGARRA NAVARRO, J. G., \& SÁNCHEZ POLO, M. T. (2001). "El cambio organizacional en el sector". Gaceta óptica, (342) pp. 10-14.

CEGARRA NAVARRO, J. G., \& SÁNCHEZ POLO, M. T. (2001). "Miopía estratégica: la calidad del servicio como elemento para fidelizar al paciente". Gaceta óptica, (352), pp. 1-7. 
CEGARRA-NAVARRO, J. G. (2004). "Una perspectiva dinamica del capital intelectural de las pymes de Murcia". Revista de economía y empresa, 21(50), pp. 103-119.

CEGARRA-NAVARRO, J. G. (2007). "Linking Exploration with exploitation through relationship memory". Journal of small business Management, 45(3), pp. 333-353.

CEGARRA-NAVARRO, J. G., \& DEWURST, F. W. (2006). "Linking shared organisational context and relational capital through unlearning: an initial empirical investigation in SMEs". The learning organization, 13(1), pp. 49-62.

CEGARRA-NAVARRO, J. G., \& RODRIGO-MOYA, B. (2005). "Learning facilitating factors of teamwork on intellectual capital creation". Knowledge and Process Management, 12(1), pp. 32-42.

CEGARRA-NAVARRO, J., \& SÁNCHEZ-POLO, M. (2008). Linking the individual forgeting context with customer capital from a seller's perspective. "The journal of the Operational Research Society", 59(12), pp. 1614-1623.

DBK. (2009). Óptica (Mercado Ibérico). Madrid: Informa D\&B.

DBK. (2010). Distribución de Óptica. Madrid: Informa D\&B.

DBK. (2011). Principales conclusiones. Óptica (Mercado Ibérico). Madrid: Informa D\&B.

DBK. (2012). Principales conclusiones. Distribución de óptica. Madrid: Informa D\&B.

DBK. (2014). Óptica (Mercado Ibérico). Datos de síntesis. Madrid: Informa D\&B.

DBK. (2015). Distribución de óptica. Datos de síntesis. Madrid: Informa D\&B.

DBK. (2016). Óptica (Mercado Ibérico). Madrid: Informa D\&B.

DBK. (2017). Óptica (Mercado Ibérico). Madrid: Informa D\&B.

DEWHURST, F. W., \& CEGARRA-NAVARRO, J. G. (2004). "External communities of practice and relational capital". The learning organization, 11(4/5), pp. 322-331.

ESPAÑA. (2013). Real Decreto Legislativo 1175/1990, de 28 de septiembre, por el que se aprueban las tarifas y la instrucción del Impuesto sobre Actividades Económicas. Texto consolidado. Madrid: Ministerio de Economía y Hacienda. Recuperado el 01 de 04 de 2015, de http://www.boe.es/buscar/pdf/1990/BOE-A-1990-23930-consolidado.pdf

EUROSTAT. (2006). NACE Rev.2 Introductory guidelines. EUROSTAT. Recuperado el 1 de 10 de 2012, de http://ec.europa.eu/eurostat/documents/1965800/1978839/ NACEREV.2INTRODUCTORYGUIDELINESEN.pdf/f48c8a50-feb1-4227-8fe0935b58a0a332

EUROSTAT. (2008). CPA 2008 Structure and explanatory notes. Luxembourg: Eurostat. Recuperado el 12 de 02 de 2013, de http://www.ine.es/clasifi/notascpa08.pdf

EUROSTAT. (2012). PRODCOM. Lista 2013. Luxembourg: Eurostat . Recuperado el 12 de 03 de 2014, de http://www.ine.es/daco/daco42/encindpr/lista_prodcom.pdf

EUROSTAT. (2017). Statistic on the production of manufactured goods. Recuperado el periodo de mayo a noviembre de 2015, de http://ec.europa.eu/eurostat/web/prodcom/ data/database/

GÓMEZ, G. (2008). "Facturación del sector óptico". Gaceta Óptica (425), pp. 66-67.

GÓMEZ, G. (2009). "El sector óptico en 2008". Gaceta Óptica (436), pp. 54-56.

GÓMEZ, G. (2010). "El sector óptico en 2009". Gaceta Óptica (447), pp. 74-78.

GÓMEZ, G. (2011). "El sector óptico en 2010". Gaceta Optometría y Óptica Oftálmica(458), pp. 52-58.

GÓMEZ, G. (2012). "El sector español de la óptica y la optometría en 2011". Gaceta de Optometría y Óptica Oftálmica (469), pp. 62-66. 
GÓMEZ, G. (2013). "El sector español de la óptica oftálmica en 2012". Gaceta óptica y optometría (483), pp. 56-61.

Hawkers revoluciona la industria de gafas de sol. (2015). El economista.

IBÁÑEZ, E. (2009). "Estudio sobre la rentabilidad de las lentes de contacto en los establecimientos de óptica y optometría (Segunda parte)". Gaceta Óptica (441), pp. 4246.

IBÁÑEZ, E. (2012). "En España hay 2,5 millones de usuarios de lentes de contacto ¿Puede seguir creciendo esta cifra?" Gaceta Óptica (471), pp. 60-64.

INE. (2003). CNAE- 93 Rev.1 Notas explicativas. Madrid : INE. Recuperado el 4 de 09 de 2012, de http://www.ine.es/clasifi/cnae93_notas.pdf

INE. (2012). CNAE-2009. Madrid: INE. Recuperado el 04 de 09 de 2012, de http://www.ine.es/daco/dac042/clasificaciones/cnae09/notasex_cnae_09.pdf

INE. (2017). Encuesta de uso de TIC y Comercio Electrónico (CE) a las empresas.

INE. (2017). Encuesta Industrial de Productos (2008-2016).

MARTÍNEZ, L., \& MONSERRAT, J. (2010). "Evaluación heurística de las web de franquicias en el sector Óptica y Optometría". Heuristic Evaluation of Websites from Optic \& Optometrist Franchises (13), pp. 1-18.

MARTíN-GUZMÁN, P. (2016). "Desafíos actuales en la estadística oficial". Estudios de Economía Aplicada, 34(3), pp. 501-530.

Mora, C. M., \& Lucas, F. M. (2017). "La estrategia de retorno de la industria española: El caso del sector calzado en Alicante, su importancia y determinantes". Estudios de Economía Aplicada, 35(3), pp. 777-800.

NICHOLS, J. J., WILLCOX, M. D., BRON, A. J., BELMONTE, C., CIOLINO, J. B., CRAIG, J. P., A., D. (2013). The TFOS International Workshop on Contact Lens Discomfort: Executive Summary. nvest. Ophthalmol. Vis. Sci., 54(11). doi:10.1167/iovs.13-13212

RODRIGO MOYA, B., \& CEGARRA NAVARRO, J. G. (2003). "Tipología de las empresas que conforman el sector óptico". Gaceta óptica (369), pp. 34-38.

SABI de Bureau Van Dijk. (2015). Sistema de Análisis de Balances Ibéricos. Recuperado el periodo de marzo a junio de 2015.

SABI de Bureau Van Dijks. (2014). Sistema de Análisis de Balances Ibéricos. Recuperado el periodo de enero a marzo de 2014.

SANTODOMINGO-RUBIDO, J., VILLA-COLLAR, C., \& MORGAN, P. (2017). "Lentes de contacto adaptadas en España en 2016". Gaceta Optometría y Óptica Oftálmica, (522), pp. 64-71.

SANTODOMINGO-RUBIDO, J., VILLA-COLLAR, C., GILMARTIN, B., \& GUTIÉRREZORTEGA, R. (2009). "Myopia Control with Orthokeratology Contact Lenses in Spain (MCOS): Study Design and General Baseline Characteristics". Journal of Optometry, 2(4), pp. 215-222.

VISIÓN Y VIDA . (2003). Libro blanco de la visión en España 2003. Madrid : ICM.

VISIÓN Y VIDA. (2006). Libro blanco de la visión en España 2006. Madrid: ICM.

VISIÓN Y VIDA Y FEDAO. (2009). Libro blanco de la visión en España 2009. Madrid: ICM.

VISIÓN Y VIDA Y FEDAO. (2013). Libro blanco de la visión en España 2013. Madrid: Grupo ICM Comunicación. 